\title{
Factores que limitan el éxito de emprendimientos turísticos comunitarios: Caso "Islas y Misterios del Titicaca"
}

\author{
Factors that limit the success of community tourism ventures: Case "Islas y \\ Misterios del Titicaca“
}

\section{Camila Elizabeth Rojas Gareca \\ camilarojasgareca@gmail.com \\ https://orcid.org/0000-0002-0546-9284}

Universidad Mayor de San Andrés, La Paz - Bolivia

\author{
Susan Pantelís \\ susanpantelis@gmail.com \\ https://orcid.org/0000-0002-3770-519X \\ Universidad Mayor de San Andrés, La Paz - Bolivia
}

Artículo recibido en enero 2021 / Arbitrado en febrero 2021 / Aceptado en mayo 2021 / Publicado en julio 2021

RESUMEN El objetivo del artículo fue identificar los factores que limitaron el éxito del emprendimiento turístico "Islas y Misterios del Titicaca" del municipio de Puerto Pérez, Bolivia. Bajo un paradigma naturalista se emplearon las siguientes técnicas de recolección de información: censo a los socios y familias involucradas; grupo focal a los representantes de turismo de cada comunidad; entrevista a entidades públicas y empresas privadas involucradas y observación en el sitio. Como resultado se identificaron seis factores que limitaron el éxito del emprendimiento: deslinde temprano de la Organización de Gestión de Destinos (OGD) encargada del proyecto, inadecuada autogestión, conflictos de intereses entre comunarios, ausencia de liderazgo local, desconfianza y discordancia de metas del emprendimiento y de las comunidades. Se concluyó que, dada la complejidad de cogestionar emprendimientos turísticos comunitarios, es crucial asegurar la alineación de objetivos a nivel proyecto, comunidades y comunarios. Asimismo, para lograr el éxito se debería analizar los factores internos y externos del emprendimiento, los cuales inciden en el desempeño de la actividad turística.

Palabras clave: Turismo comunitarios; Factores de éxito; Liderazgo comunitario; Municipal; Gestión turística

ABSTRACT The objective of the article was to identify the factors that limited the success of the tourism entrepreneurship "Islas y Misterios del Titicaca " in Puerto Pérez, Bolivia. Under a naturalistic paradigm, the following information gathering techniques were used: census members and families associated, focus group to the tourism representatives of each community, interview with public entities and private companies involved, and observation. As a result, six factors were identified that limited the success of the venture: early demarcation of the Destination Management Organization in charge of the project, inadequate self-management, conflicts of interest between community members, lack of local leadership, mistrust and disagreement between the goals of the venture and the communities. It was concluded that, given the complexity of co-managing community tourism ventures, it is crucial to ensure the alignment of objectives at the project, community, and community members. Likewise, analyze the internal and external factors of the venture, which affects the performance of the tourist activity.

Key words: Community tourism; Success factors; Community leadership; Municipal; Tourism management 


\section{INTRODUCCIÓN}

El turismo comunitario en Bolivia está en proceso de desarrollo. Según Bustillos (2016), los desafíos del turismo comunitario están ligados a la gestión comunitaria y a los canales de comercialización del producto, factores que en la mayoría de los casos condicionan su éxito o fracaso. Así se afirma en una investigación realizada por el Viceministerio de Turismo (VMT) respecto al estado del arte del turismo comunitario en Bolivia (Viceministerio de Turismo, 2013).

$\mathrm{Si}$ bien existen muchos desafíos por delante, gracias al turismo comunitario se ha logrado desarrollar y contribuir notablemente en la diversificación de la oferta turística y la dinamización de las economías locales de las diferentes regiones del país. Debido a la importancia con relación al desarrollo social y económico de las comunidades, esta actividad por lo general es financiada por Organizaciones No Gubernamentales (ONGs), fundaciones y otras instituciones, a través de distintos planes, programas y proyectos desarrollados de manera conjunta con los gobiernos locales.

Tal es el caso del emprendimiento turístico "Islas y Misterios del Titicaca" del municipio de Puerto Pérez del Departamento de La Paz, que integra tres comunidades en su ruta; Pata Patani, Quehuaya y Pariti. Gracias al apoyo de diferentes instituciones, se implementaron diferentes obras en cada comunidad, como ser: una cafetería, un albergue y tres museos. El proyecto se creó en 2008 y funcionó en el periodo 2010 - 2013. El 2013 el flujo de visitantes se redujo en un $50 \%$ en comparación de los años anteriores y en 2014 se suspendieron actividades de forma permanente (Viceministerio de Turismo, 2013)

El objetivo de esta investigación fue identificar los factores que limitaron el éxito del emprendimiento turístico "Islas y Misterios del Titicaca” del municipio Puerto Pérez, La Paz Bolivia. Este estudio se justifica porque permite aprender de la experiencia de un proyecto turístico comunitario, identificando lecciones aprendidas para próximos proyectos relacionados con el turismo comunitarios en Bolivia y en la región andina. Asimismo, permite tomar medidas para su reactivación.

\section{MÉTODO}

El estudio se realizó bajo un paradigma naturalista que tomó como objeto de estudio la implementación y puesta en marcha del emprendimiento "Islas y Misterios del Titicaca" del municipio Puerto Pérez. Se plantea como unidad de análisis a los actores que estuvieron involucrados en su implementación entre 2008 y 2013 y que actualmente perciben los factores que fueron determinantes del declive y los efectos resultantes. Las técnicas empleadas para la recolección de la información fueron: (1) censo a los socios miembros y familias involucradas de las comunidades de Pata Patani, Quehuaya y Pariti; (2) Grupo focal a los representantes de turismo de cada comunidad; (3) entrevistas semiestructuradas a los representantes de entidades públicas y empresas privadas involucradas y; (4) observación.

En primera instancia se censó a los socios del emprendimiento: en la comunidad de Pata Patani se identificaron 10 socios, en Quehuaya se identificaron 10 socios y en Pariti se identificaron a 5 socios. El $64 \%$ de los socios tenían entre 31 y 60 años en el año 2008 (año de implementación del emprendimiento). Asimismo, se realizó un relevamiento de información mediante grupos focales con la participación de los representantes de turismo de las tres comunidades en fecha 29 de abril del año 2018 durante la 1ra Feria de Bioculturas en el municipio de Puerto Pérez. Por otra parte, durante los meses de julio y agosto de 2018 se consultó mediante encuestas a las familias que trabajaron activamente en el emprendimiento, con el fin de conocer su percepción sobre las dificultades por las que atravesaron durante su operación.

Se mantuvieron entrevistas con representantes de ámbitos privado, público y ONGs, según se detalla a continuación:

Jannet Simbron, gerente general de Bolivia Milenaria y experta en temas de turismo 
comunitario. Reconocida a nivel nacional por aportes a la actividad turística de tipo convencional y comunitario. Bolivia Milenaria estuvo involucrada en la creación del emprendimiento Islas y Misterios del Titicaca, postuló el proyecto la Organización No Gubernamental Swiss-Contact en busca de financiamiento. Desde ese entonces y durante unos años Simbron estuvo a la cabeza del proyecto.

Paula Quiroga, encargada de la Fundación de Organización de Gestión de Destinos (OGD). Esta organización fue creada por la Swiss Conctact como representante regional de los emprendimientos de turismo comunitario, encargada de fortalecerlos mediante la promoción del desarrollo del turismo sostenible en los departamentos de La Paz y Beni. Trabajó activamente en el emprendimiento colaborando con la promoción y capacitación.

Isaac Callizaya, oriundo de la comunidad de Pariti, desempeñó varios cargos dentro del municipio de Puerto Pérez, actualmente se desenvuelve en el área de Culturas y Turismo como encargado oficial, quien dedicó varios años de su vida al desarrollo de la actividad turística.

Durante las visitas a las comunidades, se aplicó la técnica de la observación para registrar el estado de la infraestructura turística y otros factores relacionados con el turismo.

\section{RESULTADOS}

En este apartado se presentan los resultados de la consulta a los actores involucrados en el emprendimiento Islas y Misterios del Titicaca. Se determinaron: factores sociales, administrativos, que influyeron en el declive del emprendimiento y; cambios suscitados.

\section{ÁMBITO SOCIAL}

Mediante la consulta a los socios se determinó el grado de importancia que ellos asignan a la actividad turística: $75 \%$ considera al turismo una actividad muy importante en sus vidas y en sus comunidades, $17 \%$ considera importante y un $8 \%$ de piensan que el turismo no tiene relevancia. Profundizando en el tema, mediante grupos focales, se identificaron las siguientes dificultades preliminares: insuficientes recursos económicos, abandono de la Organización de Gestión de Destinos (OGD), falta de un responsable en turismo, diferencias entre las familias involucradas, falta de promoción, migración de los jóvenes, inexistente apoyo del municipio. Con base en este listado se consultó el grado de importancia que asignan a estos factores las familias que trabajaron activamente en el emprendimiento (según Tabla 1).

Tabla 1. Importancia percibida por las familias involucradas (1 mayor, 7 menor).

\begin{tabular}{cccc}
\hline Dificultad & $\begin{array}{c}\text { Comunidad } \\
\text { Pata Patani }\end{array}$ & $\begin{array}{c}\text { Comunidad } \\
\text { Quehuaya }\end{array}$ & $\begin{array}{c}\text { Comunidad } \\
\text { Pariti }\end{array}$ \\
\hline Insuficientes recursos económicos & 6 & 6 & 7 \\
Abandono de la OGD & 2 & 3 & 2 \\
Necesidad de un representante de turismo desde la comunidad & 3 & 2 & 1 \\
Diferencias entre las familias involucradas & 5 & 1 & 3 \\
Insuficiente promoción & 1 & 4 & 5 \\
Migración de los jóvenes & 7 & 7 & 6 \\
\hline Insuficiente apoyo del municipio & 4 & 5 & 4
\end{tabular}


Camila Elizabeth Rojas Gareca y Susan Pantelís

Como se observa en la Tabla 1 , las tres dificultades de mayor impacto responden a un patrón similar, aunque no tienen el mismo orden de importancia de acuerdo con la perspectiva de cada comunidad. Se realizó el análisis profundo de los cuatro factores más relevantes.

\section{Abandono de la OGD encargada del proyecto}

Si bien la OGD La Paz-Beni culminó su acción dentro de los plazos establecidos, la relación entre la comunidad y la organización se caracterizó por una serie de desencuentros de ideas de gestión del emprendimiento. Las familias de las tres comunidades hablan de un "abandono" por parte de esta organización. Técnicamente no hubo abandono, la OGD culminó con su gestión, aunque no con los resultados esperados.

Según el argumento de los comunarios, ellos todavía necesitaban la asistencia técnica que les brindaba la organización en cuanto al manejo del emprendimiento y por esta razón no se pudo encontrar el equilibrio que se necesitaba para mantener su sostenibilidad, provocando el declive.

Necesidad de un representante de turismo desde la comunidad

La inexistencia de un responsable de turismo como parte fiscalizadora de las comunidades afectó la coordinación con las instituciones relacionadas. Por lo general, en las comunidades del municipio de Puerto Pérez, la responsabilidad de la actividad turística está a cargo de los secretarios generales (autoridad originaria) de cada población, éstos asumen un compromiso parcial con el turismo comunitario ya que como autoridades originarias deben asumir también otras responsabilidades.

Durante la puesta en marcha, sí bien de alguna forma existieron responsables de turismo en las comunidades relacionadas con el emprendimiento, no existió un responsable a nivel general, la responsabilidad se repartía entre todos los socios del emprendimiento. No obstante, si existieron varias personas a cargo del turismo de manera esporádica. Al principio estos cargos eran asumidos por elección de las autoridades de cada comunidad. El tiempo de permanencia en todos los casos fue corto, especialmente porque no recibían compensación económica por el trabajo, por lo que las personas que asumieron esta responsabilidad rápidamente desistían del cargo.

Al no existir un responsable oficial a cargo de la actividad turística dentro del emprendimiento se perdió la mirada colectiva, cada responsable entraba con un enfoque diferente al anterior para direccionar el emprendimiento, perjudicando así el logro de los objetivos de largo plazo.

\section{Diferencias entre las familias involucradas}

Según expresan las familias, existía una constante rivalidad entre las personas que conformaban el emprendimiento y las personas que no, entorpeciendo así la coordinación. El principal motivo de rivalidad fue la distribución de los ingresos económicos que se generaban por la actividad turística. Los comunarios de Quehuaya y Pariti suponían que las personas que trabajaban en el emprendimiento ganaban más dinero que los agricultores o pesqueros de las comunidades, hecho que "no era justo" porque los trabajadores del emprendimiento percibían ingresos a costa de los atractivos turísticos del municipio; pedían que los ingresos fueran distribuidos de manera igualitaria a todos los comunarios.

\section{Insuficiente promoción turística}

Los socios aseguran que uno de los inconvenientes más grandes que fue la falta de promoción. De acuerdo con su testimonio, ellos contaban con los atractivos turísticos, la infraestructura necesaria, capacitaciones impartidas por las diferentes instituciones que intervinieron en el emprendimiento, pero la cantidad de turistas fue inferior a la expectativa. Argumentan, especialmente la comunidad de 
Pata Patani, que la promoción a nivel nacional e internacional debió estar a cargo de la OGD, las diferentes instituciones públicas como el VMT y el municipio mismo.

Desde la perspectiva de los expertos consultados los factores sociales que se valoraron son: liderazgo local, intereses y confianza. Según se detalla líneas abajo.

\section{Liderazgo local}

Las personas que asumían el cargo de administradores lo hacían de manera voluntaria y no remunerada. Si bien se les dio ésta responsabilidad, no estaban involucrados en su totalidad debido al cambio constante de autoridades locales y desconfianza respecto al manejo de los ingresos por turismo. Por tanto, se experimentaron constantes abandonos de funciones, en este contexto se designaron estas tareas a los secretarios generales de las comunidades, quienes priorizaban otras necesidades, dejando de lado las labores turísticas.

Al respecto, un ex responsable de turismo expresó: "la gente me ha dicho que me agarro el dinero y ya no quiero saber, la comunidad primero trabaja, después abandona y a los que se quedan nos señalan como que nosotros somos los únicos, luego nos aburrimos y lo dejamos". Este relato respalda la problemática.

De acuerdo con Loayza (2013), existen 3 formas diversas de participación de las comunidades en los emprendimientos comunitarios; la participación en colaboración, la cogestión y la autogestión.

En el caso de "Islas y Misterios", en una primera instancia la población local actuaba de manera participativa en la colaboración con la OGD (cogestión), una vez terminada las labores de esta organización se cedió la gestión a las comunidades, frente a los problemas previamente expuestos, se contó con el apoyo de la tour operadora Bolivia Milenaria, con el objetivo de asistir a las comunidades en cogestión para administrar el emprendimiento, infortunadamente ésta no pudo ser efectiva debido a las diferentes perspectivas que tenían las comunidades y la empresa.

\section{Intereses}

Otro de los factores internos que afectó el manejo integral del emprendimiento fueron los intereses individuales y colectivos que se antepusieron a las funciones del emprendimiento.

Los comunarios generaban mayores ingresos económicos con la agricultura, la pesca, el contrabando y otras actividades ilícitas, por lo que la actividad turística no representaba una necesidad para las personas, es por esta razón que trabajar en el emprendimiento no era de utilidad para los individuos.

Los intereses colectivos están relacionados con los intereses que existe entre las comunidades, cada una de ellas vela por beneficio propio, trabajan por separado con diferentes operadoras de turismo, perdiendo así la esencia del turismo comunitario: empoderamiento de los actores locales a través de la gestión.

La cultura y la tradición también figuran como parte de los intereses colectivos, la cosmovisión andina que se tiene en la parte del Lago menor del Titicaca está muy arraigada a estos pueblos, supera el compromiso que se tiene con el emprendimiento. Por ejemplo, en fiestas patronales la gente asistía a los eventos incumpliendo sus obligaciones con la actividad turística.

\section{Confianza}

Según los expertos, fue difícil para aquellos que querían trabajar en el emprendimiento, debido a la susceptibilidad que se generó por parte de las comunidades hacia los responsables de turismo y la misma gente que trabajaba en el emprendimiento como guía, cocinero (a), etc. De la misma manera, la población en general desconfiaba de las instituciones que trabajaban juntamente con Islas y Misterios. 
Camila Elizabeth Rojas Gareca y Susan Pantelís

El caso de Quehuaya, los financiadores invirtieron en la construcción del albergue, generando mayores conflictos. La comunidad aportaba y ayudaba de manera voluntaria donando adobes y la mano de obra. Los comunarios esperaron la retribución económica de esos aportes a corto plazo, desconociendo el tiempo promedio de retorno de la inversión.

\section{ÁMBITO ADMINISTRATIVO}

En la primera fase, la gestión económica del emprendimiento estuvo a cargo de la OGD La PazBeni, quien se encargaba de distribuir el dinero a los socios. Una vez culminada la gestión de la OGD, los socios se hicieron cargo su administración.

La administración y distribución de los recursos fue distinta en cada comunidad, pero a nivel general la gente que trabajaba dentro del emprendimiento (guía, cocinero, responsables del hospedaje) recibía un porcentaje de las ganancias. El resto estaba destinado para reinversión en infraestructura, abastecimiento de insumos y mejoras en las comunidades.

En Pata Patani el $80 \%$ de los socios asegura que el total del dinero que ingresaba a la comunidad se dividía entre el número de familias que participaban dentro del emprendimiento, el 10\% mencionó que la distribución era a través de la rotación de los socios en las actividades turísticas y el restante 10\% mencionó otras modalidades. En Quehuaya el $78 \%$ afirmó que el dinero se repartía entre los socios del emprendimiento y se dejaba un monto para mejoras generales de la comunidad, y el $22 \%$ restante asegura haber tenido otro sistema para la distribución de los recursos. En la comunidad de Pariti el $100 \%$ de los socios afirma que la distribución de los recursos se realizaba a través de la rotación de las familias para la atención del museo en esa comunidad.

Según la revisión de los documentos del proyecto se verificó que el acuerdo realizado de la distribución de los ingresos en Pariti fue 30\% para gastos de mantenimiento y operaciones y $70 \%$ debía ser beneficios de la comunidad (Flores, 2013). Mientras que en las otras comunidades no figura en documentos un acuerdo para la distribución de los recursos obtenidos por el turismo.

Según informe de los expertos, si bien existió un registro de afluencia y una tarifa determinada para el ingreso, no se empleó, en consecuencia, se desconoce los montos generados durante su periodo de funcionamiento. Los expertos coinciden en que la distribución igualitaria de los recursos tuvo una influencia negativa, por esta razón se desencadenó desconfianza entre las personas y entre las comunidades.

\section{Superestructura}

Los comunarios expresan haber recibido más apoyo de OGD La Paz-Beni durante el periodo de implementación y ejecución del proyecto (el 83\% de los comunarios expresa haber trabajado solo con esta institución) el restante $17 \%$ mencionó, además, a COSUDE y SwissContact. Destacan labores de capacitación en: guiaje en las tres comunidades; en hospedaje a Quehuaya y; en cocina a Pata Patani y Quehuaya.

Sin embargo, según el testimonio de Paula Quiroga (2018), representante de la OGD, se ofrecieron cursos en gestión empresarial enfocada en la creación de objetivos, distribución de ingresos, calidad de servicios e importancia de la fidelización del cliente.

\section{Infraestructura}

Servicios básicos, el acceso a servicios es un factor crítico en el turismo boliviano, si bien en el municipio de Puerto Pérez existe cobertura de servicios básicos (Municipio de Puerto Pérez, 2016), los servicios no se extienden en todas las poblaciones del municipio, ni siquiera en las comunidades que trabajan en la actividad turística. Según informan los comunarios, los servicios básicos solo se extienden en la zona central de 
las comunidades y los servicios higiénicos no son suficientes para abastecer las necesidades de los turistas. El emprendimiento contaba con servicios básicos necesarios para su convivencia a nivel local, pero no era suficiente ni adecuado para la recepción de los turistas extranjeros.

Los atractivos que posee el municipio tienen riqueza histórica y cultural que fácilmente puede generar el desplazamiento de los turistas hasta esta localidad, pero la gestión de los museos, el albergue y la cafetería no fueron adecuados. Actualmente estos inmuebles se encuentran deteriorados debido a la falta de mantenimiento y limpieza. Se pudo constatar que dicha infraestructura está a cargo de los secretarios generales de cada comunidad, quienes durante su ausencia cierran la entrada a los museos, el albergue y la cafetería respectivamente.

Transporte y accesibilidad, el Municipio de Puerto Pérez dispone de sistemas de transporte terrestre y lacustre, según se detalla en la Tabla 2.

Tabla 2. Transporte y accesibilidad.

\begin{tabular}{cccc}
\hline Comunidad & Vías de acceso & Transporte publico directo & Horas viaje desde El Alto \\
\hline Pata Patani & terrestre/lacustre & no & 5 horas \\
Quehuaya & terrestre/lacustre & si & 4 horas \\
Pariti & terrestre/ lacustre & no & 5 horas \\
\hline
\end{tabular}

Según se presenta en la Tabla 2, Pata Patani no tiene servicio de transporte público directo, desde la Terminal Interprovincial de la ciudad de El Alto se debe tomar una movilidad con destino a Batallas, una vez en Batallas se toma un minibús hacia Puerto Pérez y desde el muelle se toma las lanchas compartidas con destino final a Pata Patani. (esta forma de transporte está disponible solo los sábados).

Quehuaya cuenta con transporte público directo, los buses salen de la terminal interprovincial de El Alto a las 14:00 y a las 17:00 horas, la carretera en la jurisdicción del municipio requiere reparación y los buses que brindan el servicio de transporte son los llamados "microbuses de la década de los años 70". Para dirigirse hacia la comunidad de Pariti se realiza el mismo procedimiento para llegar a Quehuaya, luego se contratan botes para atravesar las aguas del Lago Titicaca.

Planta turística, Pata Patani cuenta con un museo comunitario y una cafetería; Quehuaya tiene un albergue y un museo comunitario y Pariti un museo comunitario.
Señalización, el 2010 se realizó un primer trabajo de señalización de la vía caminera, éstos se dañaron y perdieron al poco tiempo de ser implementados. La OGD también realizó un trabajo de señalización, la mayor parte de los letreros ya no existen.

\section{MARCO POLÍTICO LEGAL}

La rotación anual de los secretarios generales en las comunidades provocó un doble discurso de ellos hacia las entidades que trabajaron con el emprendimiento y también hacia las familias involucradas, de la misma manera que sucedió con los responsables de turismo o administradores de cada comunidad. Las autoridades originarias entendían la importancia de la actividad turística, pero al mismo tiempo no querían involucrarse totalmente debido a un posible fracaso.

Existe un vacío legal en las normas que regulan el funcionamiento de los emprendimientos comunitarios a nivel nacional, más específicamente en el campo turístico porque no existe un 
reglamento oficial de los emprendimientos de turismo comunitario. En consecuencia, su manejo resulta ser complejo, especialmente para las entidades financiadoras. Asimismo, confunde a los comunarios porque no conocen el nivel de intervención que debería existir, sin desplazar a la gente local de su derecho de participación en la gestión y toma de decisiones.

La inexistencia de políticas locales dificultó el manejo integral del emprendimiento. A pesar de que el emprendimiento contaba con un reglamento interno con 45 artículos divididos en 9 capítulos especificando los derechos, obligaciones, sanciones y un manual de funciones de acuerdo con las diferentes áreas de trabajo; estos no se cumplieron a cabalidad y durante el último periodo de trabajo se terminó desechando los documentos, acelerando el declive de éste.

Según los expertos, la falta de promoción no fue un factor que incidió en el declive del emprendimiento, sino la falta de compromiso e interés por parte de las familias involucradas, generando una mala imagen hacia las tour operadoras, las cuales no quisieron renovar su contrato anual que habían firmado el año 2013. La intervención de la parte privada con relación a los emprendimientos de turismo comunitario es crucial, porque las empresas tour operadoras y agencias de viaje son las que conocen el mercado y generan el desplazamiento de los turistas. Las malas experiencias generadas durante los años 2010-2012 están relacionadas con la falta de aseo y mantenimiento de los precintos turísticos e inasistencia laboral por parte de la gente local, lo cual creo una brecha entre la parte privada y el emprendimiento, rompiendo las alianzas estratégicas, necesarias para las comunidades.

A continuación, se presenta una síntesis de los efectos positivos y negativos que surgieron a raíz del emprendimiento.

\section{MEJORAS PERCIBIDAS A PARTIR DE LA IMPLEMENTACIÓN DEL EMPRENDIMIENTO}

El $40 \%$ de las familias involucradas afirma que se dinamizaron las actividades tradicionales a través del turismo; $32 \%$ señaló que surgieron beneficios a través de la venta de artesanías; $12 \%$ asegura haber recibido algún tipo de donación que beneficiaron a las comunidades de manera general, por ejemplo, en el caso de Quehuaya turistas donaron material escolar para los niños y en el caso de Pata Patani se consiguió la implementación de un baño público; $16 \%$ señaló haber percibido los cambios positivos del turismo a través del intercambio cultural y crecimiento personal.

\section{EFECTOS NEGATIVOS A PARTIR DE LA IMPLEMENTACIÓN DEL EMPRENDIMIENTO}

El cambio negativo más importante para el $56 \%$ de las familias involucradas fue el desgaste de la relación entre familias que persiste en la actualidad y en consecuencia éstas no quieren trabajar de manera mancomunada, dejando de lado el motivo por el que se creó el emprendimiento: "comunidades hermanas trabajando de manera integral para mejorar el desarrollo social a través de la actividad turística".

Por otro lado, el $32 \%$ señala a la migración de personas jóvenes que fueron capacitadas para trabajar en el emprendimiento como principal consecuencia negativa, lo que generó que actualmente no exista personal suficiente capacitado para la recepción de los turistas en dichas comunidades. El 8\% manifestó que la actividad turística generó un problema de contaminación ambiental por desechos sólidos, el restante $4 \%$ fueron motivos dispersos.

Finalmente, desde una perspectiva amplia que recoge la percepción de todos los involucrados se identificaron cinco factores que limitaron el éxito del emprendimiento: deslinde temprano de la OGD encargada del proyecto, inadecuada autogestión, conflictos de intereses entre comunarios, ausencia de liderazgo local, desconfianza y discordancia entre las metas del emprendimiento y de las comunidades. 


\section{DISCUSIÓN}

El turismo comunitario es una actividad con visión integral del desarrollo sostenible. Para el desarrollo óptimo del turismo comunitario es fundamental contar con líderes locales que compartan esta visión, esto según el concepto que da Carlos Maldonado en OIT (Maldonado, 2008).

En el caso del emprendimiento en estudio, no había líderes locales comprometidos con la actividad turística en las comunidades de Puerto Pérez, por lo cual no se logró tener una mirada colectiva entre las personas y tampoco entre las comunidades. El proyecto apostó por los líderes locales bajo el denominativo de administradores, los cuales no fueron capacitados ni motivados por lo cual faltó planificar una estrategia de empoderamiento y motivación que los vincule con el emprendimiento a largo plazo, el tiempo suficiente para poder formar nuevos líderes.

Según el autor Robbins (2005), la acción de emprender está relacionada con el esfuerzo e iniciativa de un individuo o grupo de individuos para satisfacer deseos y necesidades por medio de la innovación. Así mismo, un "emprendimiento comunitario" es considerado como iniciativa propia de los actores locales, para satisfacer sus necesidades colectivas. Islas y Misterios del Titicaca fue implantada por instituciones ajenas a las comunidades y no así por la gente local.

Este punto fue importante para determinar el nivel de compromiso y seriedad que tenían los comunarios con el emprendimiento. De acuerdo con las claves que menciona Vologuer (2010), para el buen desarrollo del turismo sostenible, hace referencia a la participación de la comunidad que debe existir desde los procesos de la planificación, gestión e involucramiento en las capacitaciones de los actores locales y su conocimiento sobre turismo, factor que no se cumplió en ninguna de las comunidades, porque los intereses personales y comunales no estaba alineados con el proyecto.
El proceso de formación y consolidación de un emprendimiento turístico comunitario conlleva años para encontrar su sostenibilidad. Como referencia se puede analizar el emprendimiento turístico comunitario "Chalalan" ubicado en la comunidad de San José de Uchupiamonas en el norte del departamento de La Paz, mismo que fue inscrito como empresa el año 1992 y luego del esfuerzo en conjunto que se realizó por parte la población y las instituciones financiadoras, el año 2000 alcanzó su punto de equilibrio y partir del año 2001 los socios comenzaron a beneficiarse de las utilidades (Fernández, 2010).

Otro ejemplo es el emprendimiento de turismo comunitario "Tomarapi" ubicado en el departamento de Oruro, el emprendimiento fue inscrito como sociedad de responsabilidad limitada (SRL) el año 2001, con la participación de 25 de las 35 familias de la comunidad Caripe en calidad de socios de la iniciativa. A partir del año 2007 se logró la sostenibilidad del emprendimiento en términos económicos, socio organizativos, ambientales y técnicos. (Cooperación Alemana, 2012).

Estos dos emprendimientos de turismo comunitario fueron tomados en cuenta porque por ser empresas pioneras en Bolivia. En estos ejemplos el proceso de formación y consolidación de un emprendimiento varía entre 6 a 9 años. Volviendo al caso de estudio, las familias socias del emprendimiento esperaron la retribución de la inversión a corto plazo, al presenciar que el emprendimiento no rendía como ellos lo esperaban desconfiaron de las instituciones involucradas y desistieron rápidamente del turismo como una actividad económica complementaria para sus comunidades y abandonaron el proyecto.

En la dinámica que presenta el turismo comunitario, es de vital importancia el involucramiento y participación de la gente local, de la misma manera los beneficios económicos deben ser distribuidos equitativamente, esto fue 
establecido por varios autores que hablan sobre la sostenibilidad, turismo y desarrollo (Abastoflor, 2008; Balam, 1996; Borrega, 2010; Maldonado, 2008). La Ley General de Turismo Bolivia te Espera 292 (VMT, 2012), menciona que la distribución de los beneficios generados debe ser de manera equitativa. Entonces, el repartimiento de los beneficios económicos debe ser de manera equilibrada, pero en el caso "Islas y Misterios del Titicaca" fue diferente, porque el emprendimiento consta de tres comunidades, las cuales tienen una realidad turística y necesidades diferentes de acuerdo a su situación actual, por esta razón la distribución igualitaria de los recursos económicos no podía ser viable a la realidad del emprendimiento.

El número de habitantes y las necesidades son muy diferentes entre Pata Patani, Quehuaya y Pariti, por lo que la distribución del dinero no debería ser igual para todos, se debería apelar a la equidad y la justicia, con el objetivo de cumplir con las metas del emprendimiento en general.

Finalmente, es importante considerar que, de acuerdo con la investigación realizada por el (Viceministerio de Turismo, 2013) respecto a los factores que inciden en el éxito o fracaso de los emprendimientos comunitarios en Bolivia, "Islas y Misterios del Titicaca" fue clasificada como emprendimiento que funcionaba, pero con algunas deficiencias que sí se podían remediar. Se menciona que para el año 2013 funcionaba con problemas de por medio, haciendo referencia al escaso acceso a los servicios básicos que se distribuyen en toda la infraestructura y planta turística del emprendimiento, es decir el albergue, los museos y la cafetería.

\section{CONCLUSIONES}

A inicios del emprendimiento "Islas y Misterios del Titicaca" los comunarios se unieron para trabajar en la actividad turística y generar beneficios económicos y sociales para las comunidades participantes, pero la mirada colectiva que se tenía fue temporal y circunstancial. La gente estaba comprometida con el emprendimiento, pero rápidamente surgieron diferencias entre familias y desconfianza en el proyecto y sus participantes. El no contar con resultados económicos en el corto plazo coadyuvó al desinterés y la migración de líderes. Con un rendimiento inferior al esperado los comunarios abandonaron el emprendimiento porque lo consideraron como un fracaso. La frustración que se creó en las comunidades generó escepticismo y una actitud pasiva en cuanto al turismo comunitario.

Existió un error de fondo y que se arrastró desde la concepción misma del proyecto, la iniciativa debió nacer desde las comunidades. El primer paso para la factibilidad del proyecto debió ser comprobar que los intereses de las metas del emprendimiento estén alineados a la de los comunarios. A pesar de haber tenido una etapa previa de socialización y educación (en temas de valoración del patrimonio de los pueblos y nociones básica de turismo comunitario) no se demostró el interés suficiente para llevar a cabo la actividad turística con éxito. Además, el poco involucramiento que existió desde en las comunidades desencadenó en la ruptura de las alianzas que existía entre el emprendimiento y las empresas privadas, el abandono de funciones $y$, finalmente, el declive del emprendimiento.

Otro factor que tuvo importante relación con el cierre del proyecto fue insatisfacción con el manejo de los ingresos. El turismo comunitario promueve la retribución equitativa de los ingresos. En este caso particular se trabajó una distribución igualitaria entre las tres comunidades, sin embargo, la población y atractivos no son homogéneos. Entonces, la distribución fue igualitaria pero no equitativa.

La migración y abandono de funciones de las personas que fueron capacitadas para trabajar en el emprendimiento fue un factor social determinante. Estas personas debieron liderar la gestión e impulsar acciones conjuntas hacia el logro de un 
objetivo común. Sin embargo, faltó motivación para constituirse en líderes (se descuidaron factores como remuneración, empoderamiento, alineación de los objetivos personales con las metas del proyecto), provocando una serie de abandonos y vacíos. Las tres comunidades carecieron de líderes capaces de llevar a cabo la gestión del emprendimiento.

Dada la complejidad que significa cogestionar emprendimientos turísticos comunitarios es crucial asegurar la alineación de objetivos del proyecto, de las comunidades y de los individuos involucrados. Asimismo, es importante conocer el periodo de retorno de la inversión y el periodo de apoyo institucional que debe extenderse en caso de ser necesario.

Finalmente, es necesario tomar en cuenta estos aspectos dentro de los emprendimientos de turismo comunitario para lograr el desarrollo sostenible y empoderamiento de las comunidades locales a través de la actividad turística y de esta manera puedan ser económicamente viables, gestionen sus recursos de manera efectiva y revaloricen y recuperen sus memorias ancestrales.

\section{REFERENCIAS BIBLIOGRÁFICAS}

Abastoflor, A. (2008). Profesionalización en el manejor del Turismo Rural en Bolivia

Cooperación Alemana. (2012). Turismo en el Parque Nacional Sajama y zonas aledañas

Balam, Y. (1996). Sociología del Turismo. Mexico: McGraw-Hill/Interamericana de Mexico S.A

Borrega, R. Y. (2010). Desarrollo, Medio ambiente y Turismo Comunitario. Problemas Ambientales, Cambio Climático y Turismo - IICSTUR, 135-144
Bustillos. (2016). Experiencias del Turismo Comunitario en Bolivia: Diagnostico y Propuesta desde los Actores Directos. La Paz

Fernández, M. (2010). El espíritu emprendedor: Aspectos teóricos y caso de emprendedores en Bolivia

Flores. (2013). Informe final de capacitación de la comunidad de Pariti. La Paz.

Loayza, A. (2013). Turismo comunitario en el Lago Titicaca un análisis comparativo de su desarrollo. La Paz.

Maldonado, C. (2008). Gobiernos locales, turismo comunitarioy sus redes, Memoria de V Encuentro consultivo regional (REDTURS). Organización Internacional del Turismo. Ginebra

Municipio de Puerto Pérez. (2016). Plan Territorial de Desarrollo Integral. La Paz.

Pantelis, S. \& Rojas, C. (2019). [Entrevista con Paula Quiroga, representante de la OGD La Paz - Beni: Gestión dentro el emprendimiento turístico "Islas y Misterios del Titicaca" del Municipio de Puerto Pérez] Grabación en audio.

Robbins, S. y. (2005). Administración. México D.F.: Prentice Hall $8^{\circ}$ Edición

Viceministerio de Turismo. (2013). Estado del Arte del Turismo Comunitario en Bolivia. La Paz.

VMT. (2012). Ley General del Turismo. La Paz: Viceministerio de Turismo. Bolivia

Voleger, C. (1 de septiembre de 2010). 12 Claves del Turismo Sostenible. 12 Claves del Turismo Sostenible. Santiago, Chile 